\title{
CONTENT BASED BATIK IMAGE CLASSIFICATION USING WAVELET TRANSFORM AND FUZZY NEURAL NETWORK
}

\author{
Abdul Haris Rangkuti \\ School of Computer Science, University of Bina Nusantara, Jakarta, Indonesia
}

Received 2013-09-14; Revised 2013-10-03; Accepted 2013-12-19

\begin{abstract}
In this paper we introduce the content-based image classification using wavelet transform with Daubechies type 2 level 2 to process the characteristic texture consisting of standard deviation, mean and energy as Input variables, using the method of Fuzzy Neural Network (FNN). All the input value will be processed using fuzzyfication with 5 categories namely Very Low (VL), Low (L), Medium (M), High (H) and Very High (VH). The result will be fuzzy input in the process of classification with neural network method. Batik images will be processed using 7 (seven) types of batik motif which is ceplok, kawung, lereng, parang, megamendung, tambal and nitik. The results of the classification process using FNN is Rule generation, such that for a new image of batik motif types can be immediately determined after FNN classification is completed. For the level of precision, this method is between $90-92 \%$, including if we use the rule generation to determine the level precision is between $90-92 \%$.
\end{abstract}

Keywords: Batik Image, Wavelet Transform, Daubechies, Fuzzy Neural Network, Fuzzification, Rule Generation, Classification

\section{INTRODUCTION}

Batik cloth is a decorative cloth, and has an important role for conducting traditional ceremonies, in Java island as well as Indonesia. Even in several official state event where all guests are requested to wear batik apparel. Basically batik is an Indonesian cultural heritage that has been very popular in almost all over the world. However, there are a few batik patterns that are special and it is repeated many times in the design. The patterns can be used as a feature/characteristic in order to identify and to explore the origin of the batik cloth such as texture, color and shape (Fig. 1) as the batik pattern characteristic.

Basically, the process to retrieve the batik image which is based on low-level feature (characteristic) is an area that is mostly under research and there are still many to be explored inn image processing. This is also to anticipant the storage of the images and retrieval of image from a collection of images become optimal, fast and effective. One of the approaches that will be used is
Query by Example, which is one derivative from the development of CBIR (Content Base Image Retrieval). The target of this method is to handle the limitation of texts against the method of image retrieval, which is subjective because the human-created keywords, and very expensive because of manual processing. The content based image classification is an important task in terms of indexing and image retrieval. Due to this reason the image retrieval concept based on content is the best retrieval based on human concept (Rangkuti et al., 2012a; Margaretha et al., 2009).

Images with different textures have different characteristics. Relative to batik image, texture feature is important because the ornament in the cloth can be viewed as different texture composition. On top of that, the batik pattern can be influenced by the shape characteristics, which is what batik is composed of. For example, patterns of slopes with slanted lines, machete shaped pattern with slanted lines that has a dent on each top and bottom and other patterns. This research will extend knowledge to understand batik patterns based on characteristics of the batik texture. These characteristics are the base for conducting image classification. 

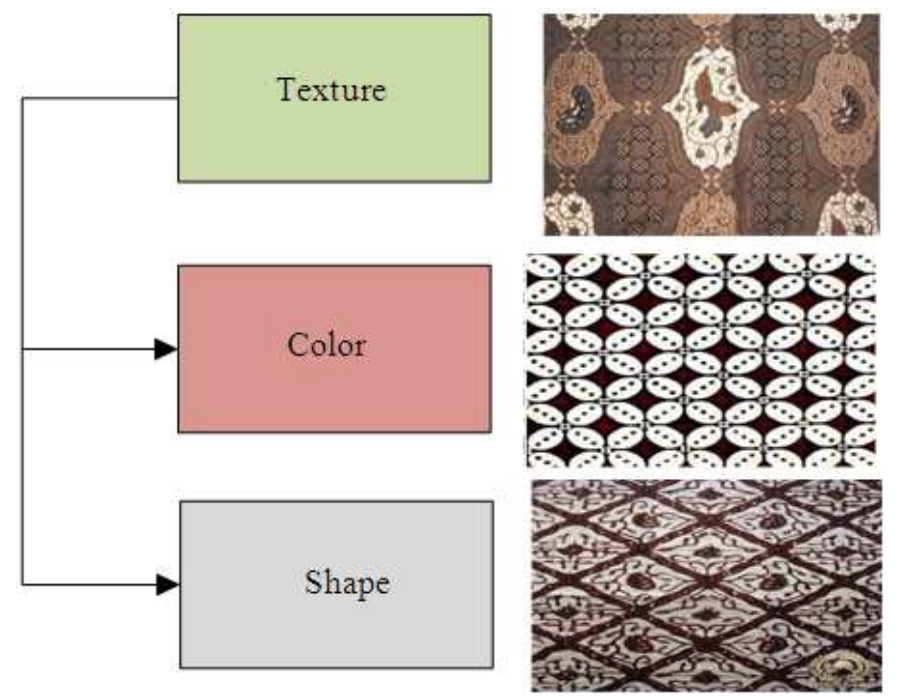

Fig. 1. The batik pattern characteristic explored on the texture, color and shape

There is a couple of research that focuses on image classification process based on texture, especially of images. Several researches use neural network in conducting classification, Neural network for classification is preferable because of its ability to process in parallel and the ability to perform decision making (Ajay et al., 2012). Besides, in order to increase the ability to group or classify content based images, supporting approaches are also used such as Fuzzy Logic and Wavelet Transform This research focuses on batik image, which is already under research a starting a couple of years ago, although it is using a similar method to other general classification method (Rangkuti et al., 2012b).

In previous researches, there exist some weaknesses on the result, where the accuracy level to grouping or classification of batik cloth is still less than $90 \%$. Also in terms of batik pattern under research is still too few. Therefore the picture of batik pattern complexity is not represented clearly. The target of this research is to obtain the effectiveness in conducting batik pattern classification based on the similarity of the pattern, whether it is more accurate or more relevant towards its image classification. Therefore it is easier to recognize the origin of the batik including the associated traditional story behind each and every single pattern of batik.

\subsection{Objectives and the Significance of Research}

The objective of this research is:

- To develop an effective concept and methodology for image classification process on batik that will be defined and classified as a group. Batik image will be classified based on the pattern and the result will determine the classification of the batik pattern

- To improve the performance of image classification based on is pattern, especially on batik. Because of the development of rules, the classification process simpler

- To process CBIR on batik images efficiently and effectively based on the classification to become 7 categories

The urgency significance of this research is as follows:

- To improve the ability to identify the batik image pattern, so that it can provide more information about the type of batik accurately

- To improve batik retrieval performance using Fuzzy Neural Network classification process

- To be used as a reference in conducting image retrieval of image characteristic based on the similarities that is developed in the field of bioinformatics and cultural information

\subsection{SCOPE}

The scope of this research is as follows:

- To research on batik image type with the image format .JPG. PNG, TIFF, BMP

- The batik image classification is conducted $n$ texture patterns which is divided into 3 variables: Mean, standard deviation and energy 
- Batik image classification process is based on pattern using Fuzzy Neural Network technique

- To analyse texture characteristic on batik pattern using wavelet transform and shapes using Invariant Moment

- The use of background colour is not the main reason

- The texture characteristic of the batik image will to have any effect on the position and location of the image

- Can be affected by image noise

Basically this research is focused on some batik patterns with a specific image texture and shape. This research can be developed on the characteristic of other image such as Natural Phenomenon, Forest Condition, wayang shapes, paintings and candi (cultural Informatics).

\subsection{The Output of this Research}

- The result created by fuzzyfication on all batik images that has been processed. There are 5 categories which is Very low, Low, Middle, High and Very High

- An accurate percentage is obtained from the classification of 7 baik patterns based on learning data and test data

- The development of rule generation while detecting a baik pattern, based on segmentation of image shapes which is simpler, but having an excellent performance

In this research there are 7 types of batik patterns, suach as "ceplok", "'lereng", "parang", "Megamendung", "kawung", "nitik" dan "tambal". With calassificatio process on patterns, it is easier to retrieve using the similar charactereristics and this can be performed in future (Fig. 2) in general the similar pattern that is still researched.

\subsection{Literature Study}

\subsubsection{Previous Research}

Basically, there are other research developing the classification concept using neural network and fuzzy logic. This research is performed while developing the CBIR concept on images, and increasing the accuracy percentage. As time goes by the utilization of this concept becomes more and improved by the development of new methods, including developing new methods that include combination of certain methods to improve the precision, where each and every image that is observed is classified based on the characteristics.
Some research proposed image classification method such as content based classification using neural network and fuzzy logic. Other implementation than medical image classification is a technique for assigning a medical image to a class among a number of image categories. Due to computational complexity, it is an important task in the content-based image retrieval (Pourghassem and Ghassemian, 2011). Usually the conventional method in medicine for brain MR images classification and tumor detection is by human inspection. Fuzzy logic technique is more accurate but it fully depends on expert knowledge, which may not always available. Here we extract the feature using PCA and after that training using the ANFIS tool. The performance of the ANFIS classifier was evaluated in terms of training performance and classification accuracy (Bhardwa and Siddhu, 2013).

A fuzzy Neural network (FNNs) also provide a new approach to classify multispectral data and to optimize classification rules. Neural network handles problems at numerical level, whereas fuzzy is related to it from semantics level or linguistic level. NNs synthesize fuzzy logic and neural network (Kulkarni and McCaslin, 2006).

The Classification Method is conducted using group approach using the concept of neuro fuzzy. By using neuro-Fuzzy approach to classify image based on content with the retrieval of $2 \mathrm{D}$ images and using wavelet transformation (Balamurugan and Anandhakumar, 2009). The implementation of classification process on wood pattern as a pencil maker is also developed using a new approach which is neuro-fuzzy logic. This new approach simplifies process in terms of obtaining wood pattern (output) effectively and at lower cost (Franca and Gonzaga, 2010). A new approach for image classification based on the color information, shape and texture is presented. Where they use the three RGB bands of a color image in RGB model to extract the describing features (Lotfi et al., 2009).

This research proposes a step of batik image introduction using several methods and at the same time producing a classification of the batik image where the precision value has been calculated based on training data and test data. Based on the above problem and some research on image processing, our research will design a classification concept to support batik image retrieval that has specific ornaments and patterns, unique and many varieties. By implementing fuzzy logic query for searching such as query process using query with images based on texture characteristics from the batik image database. Some researches that used fuzzy logic in performing classification, generally used for image characteristic representation, where performance of classifying the image is improved. 

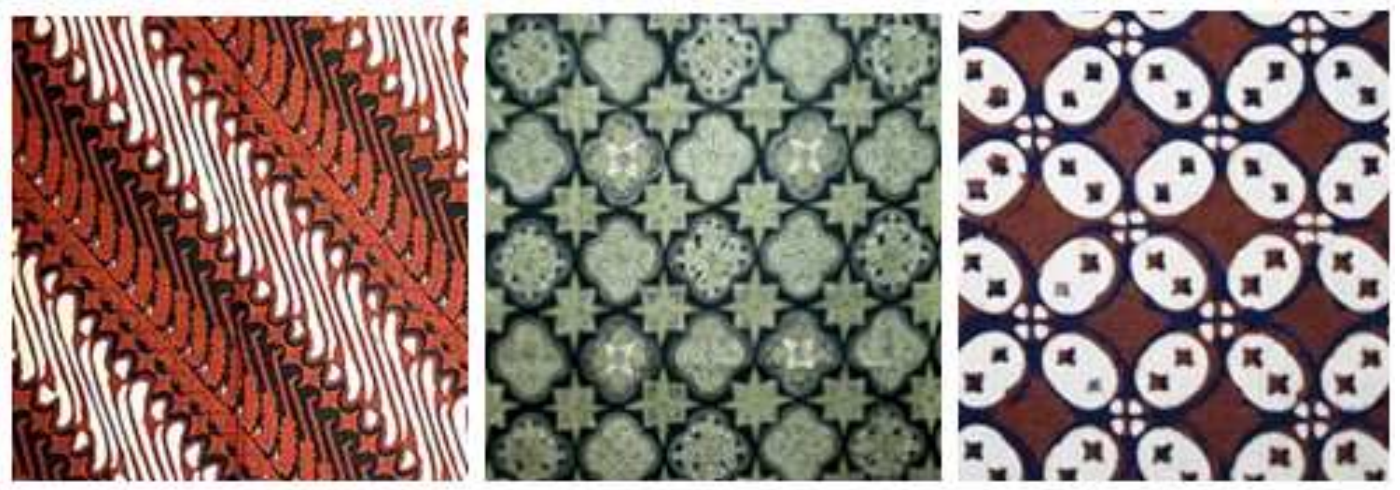

Fig. 2. Some of Batik pattern which is still researched

The researchers that is related to batik image is using conventional CBIR concept, where for image retrieval becomes relevant to sample images, the batik pattern recognition allow the approach to become more flexible using Generalized Hough Transform. This method is to recognize some patterns within the same collection of images (classification) (Sanabila and Manurung, 2009). The classification concept showed that performing classification with cluster batik image, based on patterns, contrast and supporting colour, where the colour and contrast is supported by the supporting algorithm (Margaretha et al., 2009; Moertini and Sitohang, 2005).

The development of this batik images generally focused on the batik image search to represent image characteristics which is texture. Some are focused on shapes and colour. For texture characteristics on certain images uses a combination of wavelet transform which is Discrete Wavelet Transform and Rotated Wavelet Filter. This method is developed for a specific batik image, also for the CBIR concept using Canberra Distance, whereas the batik classification process is using multi-layered preceptron (Kokare et al., 2007). As for other methods, it is using batik image construction using codebook and framework keyblock shows that for batik pattern classification based on texture characteristics is using packet wavelet transformation (Wahyudi et al., 2009).

\subsection{Wavelet Transform Method}

Wavelet is a mathematical function that helps to elaborate the original image into an image in the frequency domain, which then is able to be divided into different subband frequency components. Every component is studied with resolution associated with a scale. Wavelet transformation has an advantage compared to traditional Fourier method, in terms of physical analysis, where the signal contains discontinuation and steep curve. This paper presents characteristic extraction and batik image classification using Daubechies 2 wavelet transform and neural reverse propagation. The Wavelet feature is obtained from the original image texture and the associated supporting images. The feature consists of different combination of the sub-band images that offers a better strategy to differentiate image classification and improve the classification level (Ajay et al., 2012).

Discrete Wavelet Transform (DWT) is grouped into two which is DWT forward and DWT reverse. At DWT forward, image data is decomposed, starting by decomposing rows followed by decomposition operation for columns on the image coefficient at the output of the first step. The input image is interpreted as signal, decomposed using Lo_D (Low Pass Filter Decomposition) and Hi_D (High Pass Filter Decomposition) and then second down sampling is perfumed. The output is a low frequency signal and high frequency signal. The two process described previously is performed twice for each row and columns so that four sub-band will be produced containing low frequency and high frequency information. The approximation Coefficient contains background information and Coefficient detail which is: horizontal detail, vertical detail, and diagonal detail that contain edge information. The wavelet Transformation above is a wavelet transformation level 1, higher level transformation can be obtained by dividing the sub-band residue approximation coefficient into smaller sub-band. The following picture illustrate the image decomposition process, Fig. 3. 

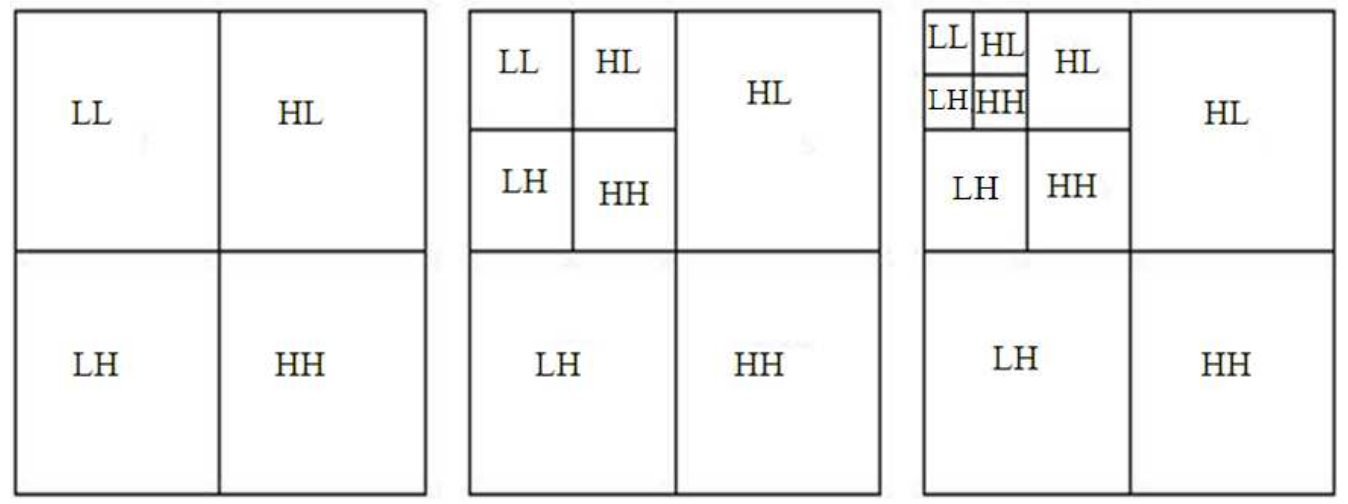

Fig. 3. Image decomposition on wavelet discrete level 1, 2 and 3

LH1, HL1, and HH1 is the result of decomposition level 1. LL2 is not shown on the diagra, because it is immediatey decomposed into LL2, LH2, HL2 and HH2. Decomposition process always start from the top left corner (Pratikaningtyas, 2010; Hazra, 2011).

\section{MATERIALS AND METHODS}

\subsection{Thinking Framework}

The thinking framework for the analysis of batik image retrieval system based on batik image similarity by implementing fuzzy logic from the Fagin algorithm, can be seen below. Basically there are 5 main steps involved in performing image classification which is:

- Collection and Selection of batik image

- Preprocessing Image

- Feature Extraction

- Classification of batik image

- Rule Generation Process

All the process will be seen in analysis step of batik image classification in Fig. 4.

\subsection{Collection and Selection of Batik Image}

At the first step, collection is performed and at the same time selecting all batik image collected. Doing the collection of batik image is done in several ways such as through the Internet, taking a digital photograph or from an image collection CD-batik, or by selecting the images manually. All the collected images have a file extension of *. jpg, *. png or *. bmp. The Batik image must also be clear in shape and texture. Some motif to be studied focused on seven types of ceplok, kawung, lereng, Parang, mega mendung., Nitik and Tambal.

\subsection{Preprocessing Image}

The second step, all data will be pre-processed by making all image to be gray-scaled. The purpose of this is to ensure that during extraction, there will be no interference due to RGB/HSV.

\subsection{Feature Extraction}

While performing Feature extraction process, it is elaborated into 3variables which is: Mean, Standard Deviation and Energy. The 3 variable above will be processed using wavelet Transform Daubechies 2 level 2. This is a new study of the characteristic texture in which the variable will be calculated using the method of wavelet transform. This variable is only focused in calculating the approximation coefficients which are part of the 4 sub-bands. There are some supporting of command such as (MATLAB):

$$
[\mathrm{C}, \mathrm{S}]=\text { wavedec2 }\left(\mathrm{i}, 2,{ }^{\prime} \mathrm{db} 2 '\right), \mathrm{A}=\operatorname{appcoef} 2\left(\mathrm{C}, \mathrm{S}, \mathrm{\prime}^{\prime} \mathrm{db} 2{ }^{\prime}, 2\right)
$$

Note: Wavedec $2=$ wavelet decomposition 2D, $\mathrm{db} 2=$ Daubechies type 2, appcoef $2=$ approximation coefficients 2D. Some support variable in the motifs of batik on the texture are:

- Wavelet Energy: $\sum_{\mathrm{i}, \mathrm{j}} \mathrm{P}(\mathrm{i}, \mathrm{j})^{2}$

- Wavelet Entropy: $-\sum_{\mathrm{i}, \mathrm{j}} \mathrm{P}(\mathrm{i}, \mathrm{j}) \log \mathrm{P}(\mathrm{i}, \mathrm{j})$

- Wavelet Standard deviation: $\left(\frac{1}{\mathrm{n}-1} \sum_{\mathrm{i}=1}^{\mathrm{n}}\left(\mathrm{x}_{\mathrm{i}}-\overline{\mathrm{x}}\right)^{2}\right)^{\frac{1}{2}}$

Description of the calculation process 3 variable characteristic texture of the approximation coefficient. The wavelet process can be seen in the Fig. $\mathbf{5}$ as below. 


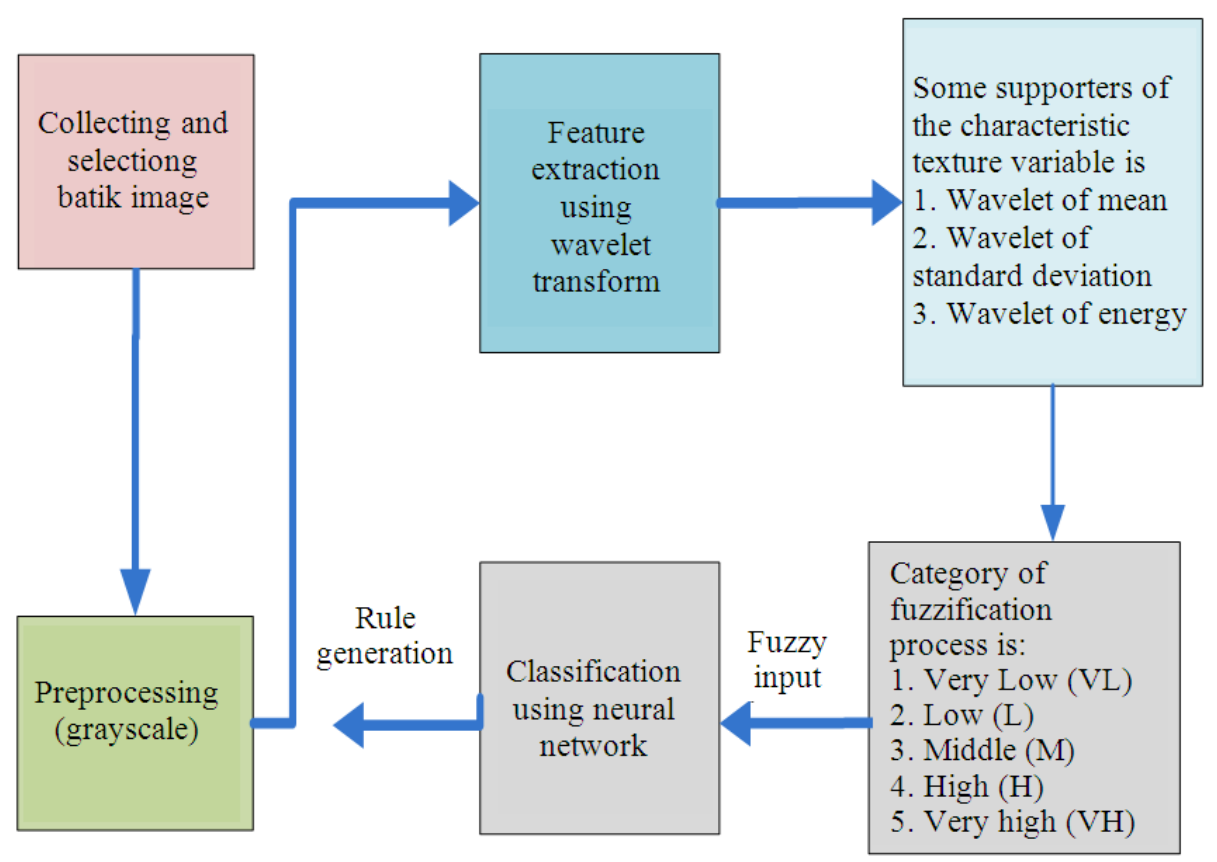

Fig. 4. Analysis step of Batik Image classification process using input from some variable

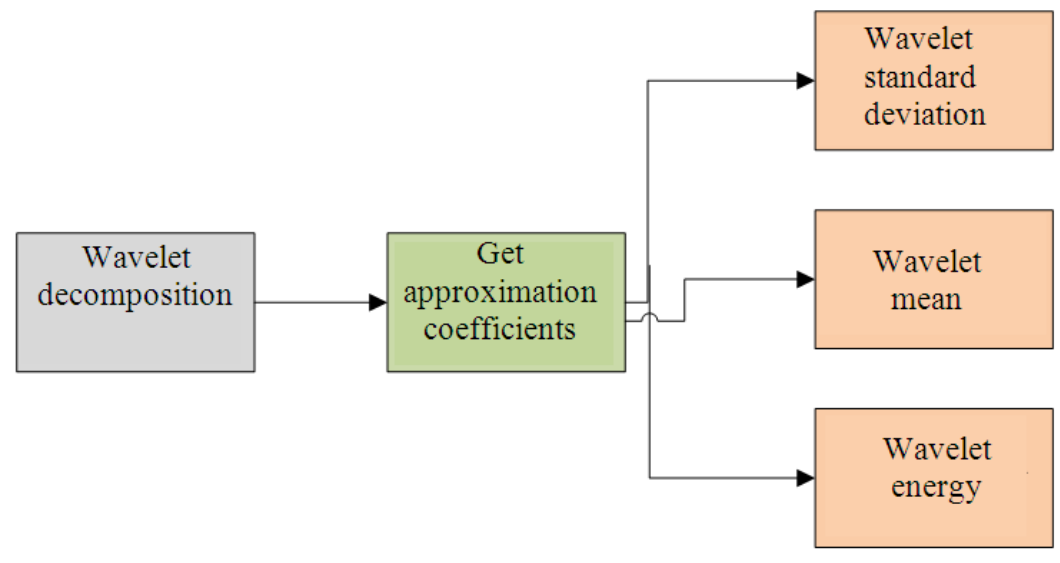

Fig. 5. The process of texture feature extraction using wavelet transform

\subsection{Classification of Batik Image}

At Layer 1, there are three crisp input which is a feature from the batik image: Energy $\left(\mathrm{X}_{1}\right)$, Mean $\left(\mathrm{X}_{2}\right)$, Standard deviation $\left(\mathrm{X}_{3}\right)$. The three crisp input will be forwarded to layer 2 to be processed by fuzzyfication in order to obtain a fuzzy input in the form of membership degree for each linguistic value. At the fuzzyfication process, every crisp inpt will be transformed into fuzzy input with 3 linguistic values for each crisp input which is very low $(\mathrm{VL})$, Low $(\mathrm{L})$, middle $(\mathrm{M})$, high $(\mathrm{H})$ and
Very High (VH). This Fuzzy input will be the input for neural network which reside between layer 2 and layer 3 . Membership function that is used in this research is the Triangle Membership Function.

Layer 3 has seven neuron to classify seven types of batik image type: ceplok (CP), kawung $(\mathrm{KW})$, parang (PR), mega mendung (MM), lereng (LR), Tambal(T), $\operatorname{Nitik}(\mathrm{N})$. Every neuron will produce an output which is a binay value " 0 " or " 1 ". The combination of seven binary numbers at layer 3 shows the class and the pattern that is input into the system. All the process of classification 
will be seen in Fig. 6. The class coding schema (target) for each batik pattern is presented in Table 1.

\section{Learning Algorithm}

The purpose of the Learning Process on FNN is to obtain an optimal network scale at the network connection between layer 2 and layer 3 .

The Learning Algorithm is described below:

Set learning rate value $(\alpha)$;

Set network scale $\left(\mathrm{w}_{\mathrm{ij}}\right)$;

i: neuron index at layer 2,

$\mathrm{j}$ : neuron index at layer 3;

While not stop confdition do:

For each learn data, perform:

1. Calculate fuzzy input for variable Energy $\left(X_{1}\right)$;

2. Calculate fuzzy input for variable Mean $\left(\mathrm{X}_{2}\right)$;

3. Calculate fuzzy input for variable Standard deviation $\left(\mathrm{X}_{3}\right)$;

While not stop confdition do:

For each learn data, perform:

1. Calculate fuzzy input for variable Energy $\left(X_{1}\right)$;

2. Calculate fuzzy input for variable Mean $\left(\mathrm{X}_{2}\right)$;

3. Calculate fuzzy input for variable Standard deviation $\left(\mathrm{X}_{3}\right)$;

For each neuron $\mathrm{j}$ at layer 3 , perform:

1. Calculate value $y_{-}$net $_{\mathrm{j}}$ at layer 3 using the formula:

$$
\mathrm{y}_{-} \text {net }_{\mathrm{j}}=\sum_{\mathrm{i}=1}^{7} \mathrm{w}_{\mathrm{ij}} \mathrm{x}_{\mathrm{i}}
$$

2. Calculate value $y(j)$ at layer 3 using the formula:

$$
y_{j}=\frac{1}{1+\exp ^{-\left(y_{-} \text {net }_{j}\right)}}
$$

3. Calculate error (E) using the formula:

$$
\mathrm{E}_{\mathrm{j}}-\operatorname{Target}_{\mathrm{j}}-\mathrm{y}_{\mathrm{j}}
$$

4. Calculate $\mathrm{MSE}_{\mathrm{j}}$ with the formula:

$$
\operatorname{MSE}_{\mathrm{j}}=\frac{\sum_{\mathrm{j}=1}^{7}\left(\mathrm{E}_{\mathrm{j}}\right)^{2}}{\text { jumlah output neuron }}
$$

5. Calculate delta rule $(\delta)$ using the formula:

$$
\delta_{j}=E_{j} y_{j}\left(1-y_{j}\right)
$$

For each scale between layer 2 and layer $3\left(\mathrm{w}_{\mathrm{ij}}\right)$, perform:

1. Calculate difference of scale $\left(\Delta \mathrm{w}_{\mathrm{ij}}\right)$ using the formula:

$$
\Delta \mathrm{w}_{\mathrm{ij}}=\mathrm{aw}_{\mathrm{ij}} \delta_{\mathrm{j}}
$$

2. Calculate new scale $\left(\mathrm{w}_{\mathrm{ij}}(\right.$ baru $\left.)\right)$ using the formula:

$$
\mathrm{w}_{\mathrm{ij}}(\text { baru })=\mathrm{w}_{\mathrm{ij}}(\text { lama })+\Delta \mathrm{w}_{\mathrm{ij}}
$$

3. Calculate the average MSE for one epoch (MSE $\mathrm{epoch}_{\text {) }}$ using the formula:

$$
\operatorname{MSE}_{\text {epoch }}=\frac{\sum_{\mathrm{j}=1}^{7} \mathrm{MSE}_{\mathrm{j}}}{\text { jumlah epoch }}
$$

\subsection{Rule Generation Process (Backtracking)}

- We use the learning data to perform feed forward process on layer 2 and layer 3 (part of neural network)

- For each data pattern on the learning data, perform the following process:

- For each neuron on the outpuut layer, find the neuron having the value $\mathrm{f}(\mathrm{net})$ of $\mathrm{y}>=$ threshold. For this case, the threshold value is set to 0.5 . This neuron is called te active neuron

- For each neuron on the input layer which is connected to the active neuron on the output layer, perform the following process:

B1: Perform the calulatio of activity level (z) using the formula: $\mathrm{z}(\mathrm{i}, \mathrm{j})=\mathrm{w}(\mathrm{i}, \mathrm{j}) \cdot \mathrm{y}(\mathrm{j})$, where, $w$ is the netwrk scale, $y$ is the output on each neuron at the output layer, $i$ is the index number for neurons at the input layer and $\mathrm{j}$ is the index number for neurons at the output layer.

B2: Next, if a neuron at the input layer has an acivitiy level (z) that is $>=$ threshold $(0.5)$, then the connection between neuron at the input layer and output layer is called the active connection. Therfore, one active neuron at the output layer will have a collection of active connection which is connected to neuron at at the input layer that has activity level (z) $>=$ threshold. One unit of active connection for one active neuron means one rule candicate.

B3: After all ata at the learning data is input into system, and with several rule candidates, then this rule candidates will be selected, removing redundant rules and contradictory rules. Rules that are contradictory is defined to have the same antisenden, but different consequences. 


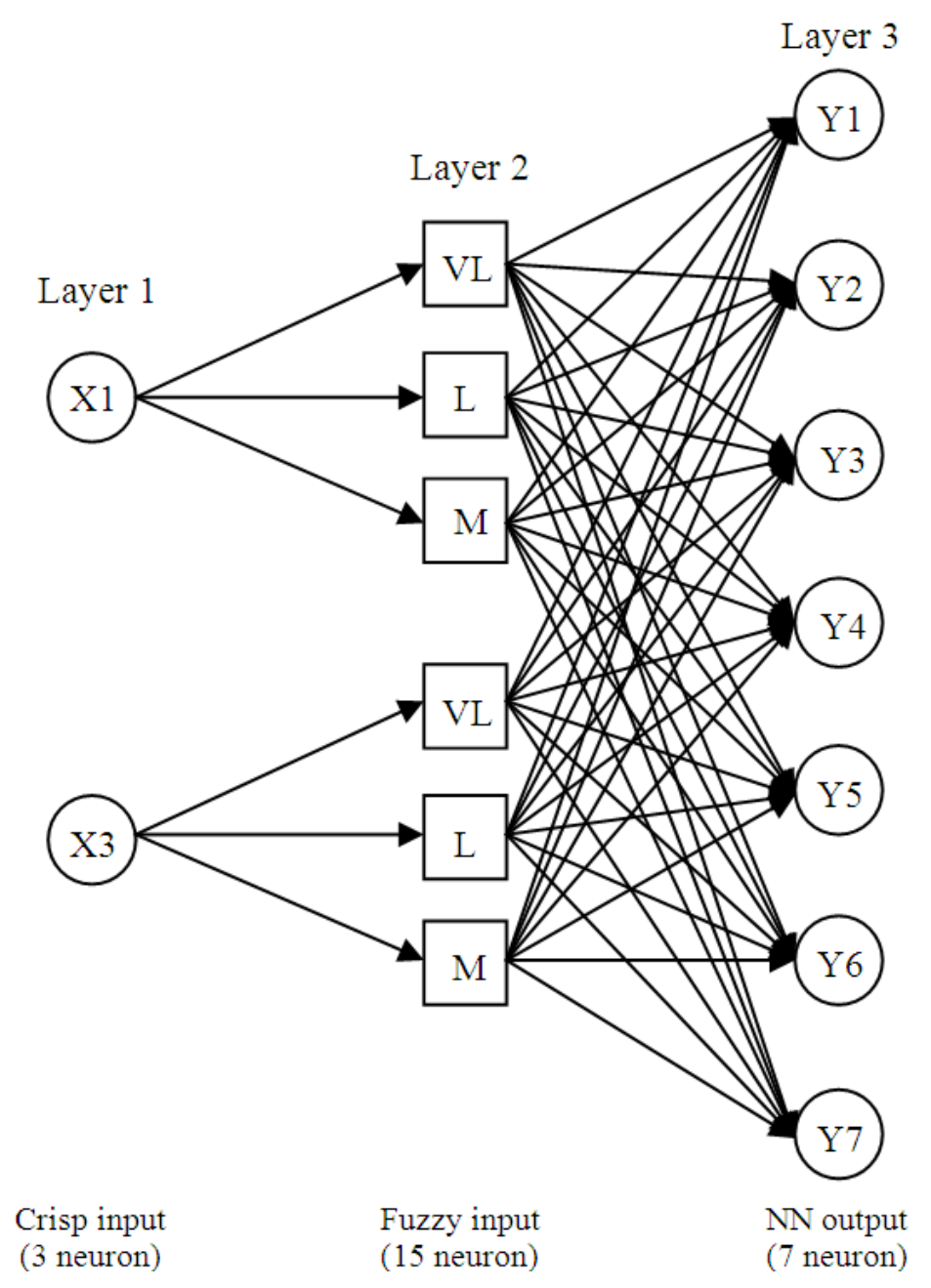

Fig. 6. Neural network architecture for batik image classification

Table 1. Target on neural network schema process on batik image pattern

\begin{tabular}{|c|c|c|c|c|c|c|c|}
\hline \multirow[b]{2}{*}{ Batik type } & \multicolumn{7}{|c|}{ Class coding schema at Layer 3} \\
\hline & Nr.1 & Nr.2 & Nr.3 & Nr.4 & Nr.5 & Nr.6 & Nr.7 \\
\hline Ceplok & 1 & 0 & 0 & 0 & 0 & 0 & 0 \\
\hline Kawung & 0 & 1 & 0 & 0 & 0 & 0 & 0 \\
\hline Lereng & 0 & 0 & 1 & 0 & 0 & 0 & 0 \\
\hline Parang & 0 & 0 & 0 & 1 & 0 & 0 & 0 \\
\hline Megamendung & 0 & 0 & 0 & 0 & 1 & 0 & 0 \\
\hline Tambal & 0 & 0 & 0 & 0 & 0 & 1 & 0 \\
\hline Nitik & 0 & 0 & 0 & 0 & 0 & 0 & 1 \\
\hline
\end{tabular}

Suppose there are two candicate rule that is contradictory of each other, rule 1: "if EA = 'Low' and

STD = 'Medium' then class is 'pattern 1'", rule 2: "if EA $=$ 'Low' and STD = 'Medium' then class is 'pottern 3', 
then to determine which rule will be used is calculated by calculating the Degree of significance (D) using the forumula: $\mathrm{D}=\mu_{0} \cdot \mu_{1}, \mu_{2} \ldots \mu_{\mathrm{n}} \cdot \mathrm{y}_{\mathrm{j}}$ where $\mu$ is fuzzy input (result from fuzzification) and $y_{j}$ is the valueobtained at the output layer. Therefore, from the above example, D can be calculated as follows:

$\mathrm{D}_{\text {(rule 1) }}=\mu_{\mathrm{EA}}($ Low $) \cdot \mu_{\mathrm{STD}}($ Medium $) \cdot \mathrm{y}_{1}$

$\mathrm{D}_{\text {(rule 2) }}=\mu_{\mathrm{EA}}($ Low $) \cdot \mu_{\mathrm{STD}}($ Medium $) . \mathrm{y}_{3}$

The Rule having the larger $\mathrm{D}$, is the rule that will be used (Kulkarni and McCaslin, 2006).

\section{RESULTS AND DISCUSSION}

On the basis of our research has been carried out, it is in the process of classification of the motif by using Fuzzy Neural Network algorithm with different structure then the percentage of similarity can be seen in Table 2.

From Table 2, the result summary represents of the research with learning data from 145 batik image, with 7 batik image pattern type. The precision value ia obtained after learning with architecture 3-15-7 which is 3 input variable input (mean, standard deviation dan Energy), 15 layer value from fuzzyfication process with each variable divided into 5 categories: Very Low, Low, Middle, High and Very High, 7 layer amount of output, and the resulted of the precision is $86 \%$. Whereas using the architecture 3-15-10-7 which is 3 input variable, 15 layer is the result of fuzzyfication, 10 hidden layer, 7 layer amount of output, produce a precision of $94 \%$.

By using different types of architecture, it can be concluded that the classification of the data testing and test data become more leverage by using a type of Architecture 3-15-10-7. Despite the increase in the number of hidden layer are used ranging from 10, 15, 20 and 25 layers. But after investigation into more leverage by using layer 10 hidden layer.

Table 2. Result summary from the batik image classification process using fuzzy neural network

\begin{tabular}{lll}
\hline MSE & $9,99 \mathrm{E}-01$ & $9,81 \mathrm{E}-01$ \\
\hline Epoch & 279.135 & 184.800 \\
Architecture & $3-15-7$ & $3-15-10-7$ \\
Learning Rate & 0,2 & 0,2 \\
$\%$ Precision & 86 & 94 \\
\hline
\end{tabular}

\section{CONCLUSION}

- In the process of batik classification algorithm using fuzzy neural network with 3 input variables are the mean, standard deviation and energy, the precision rate can reach more than $85 \%$. Even with using the neural network architecture 3-15 - 10-7 can produce precision of above $95 \%$

- Wavelet transform method Daubechies type 2 level 3 is used to calculate the characteristic texture of the input variables, which focus on the approximation coefficients. This method will result in the value of a variable that can distinguish between the types of batik motif

- In this study only focused on 8 well-known motif and other motifs will be developed in a more diverse and complicated

- Continuation of this study will be developed to identify characteristics of the image based on a more complicated and complex, such as the shape/texture of the blood disease cells, cancer cells, tumor cells, even in the field of informatics culture

\section{REFERENCES}

Ajay, K.S., S. Tiwari and V.P. Shukla, 2012. Wavelet based multi class image classification using neural network. Int. J. Comput. Applic., 37: 21-25. DOI: 10.5120/4597-6555

Balamurugan, V. and P. Anandhakumar, 2009. Neurofuzzy based clustering approach for content based image retrieval using 2Dwavelet transform. Int. J. Recent Trend Eng., 1: 47-53.

Bhardwa, A. and K.K. Siddhu, 2013. An approach to medical image classification using neuro fuzzy logic and ANFIS classifier. Int. J. Comput. Trends Technol., 4: 236-240.

Franca, C.A. and A. Gonzaga, 2010. Classification of wood plates by neural networks and Fuzzy logic. University Federal at Sao Carlos.

Hazra, D., 2011. Texture recognition with combined GLCM, wavelet and rotated wavelet features. Int. J. Comput. Electr. Eng., 3: 1793-8163.

Kokare, M., P.K. Biswas and B.N. Chatterji, 2007. Texture image retrieval using rotated wavelet filters. Patt. Recogn. Lett., 28: 1240-1249. DOI: 10.1016/j.patrec.2007.02.006

Kulkarni, A.D. and S. McCaslin, 2006. Fuzzy neural network models for multispectral image analysis. Proceedings of the 5th WSEAS International Conference on Circuits, Systems, Electronics, Control and Signal Processing, Nov. 1-3, Dallas, USA., pp: 66-71. 
Lotfi, M., A. Solimani, A. Dargazany, H. Afzal and M. Bandarabadi. 2009. Combining wavelet transforms and neural networks for image classification. Proceedings of the 41st Southeastern Symposium on System Theory University of Tennessee Space Institute Tullahoma, Mar. 15-17, IEEE Xplore Press, Tullahoma, TN., pp: 44-48. DOI: 10.1109/SSST.2009.4806819

Margaretha, E., A. Azurat, R. Manurung and A. Murni, 2009. Content-based information retrieval system for batik application. University of Indonesia.

Moertini, V.S. and B. Sitohang, 2005. Algorithms of clustering and classifying batik images based on color, contrast and motif. ITB J., 37: 141-160. DOI: 10.5614/itbj.eng.sci.2005.37.2.5

Pourghassem, H. and H. Ghassemian, 2011. Contentbased medical image classification using a new hierarchical merging scheme. Comput. Med. Imag. Graph, 32: 651-661. PMID: 18789648

Pratikaningtyas, D., 2010. Klasifikasi batik mengunakan metode transformasi wavelet. Paper Skirpsi, UNDIP.
Rangkuti, A.H., N. Hakiem, R.B. Bahaweres, A. Harjoko and A.E. Putro, 2012a. Analysis of image similarity with CBIR concept using wavelet transform and threshold algorithm. Proceedings of the IEEE Symposium on Computers and Informatics, Apr. 79, IEEE Xplore Press, Langkawi, pp: 122-127. DOI: 10.1109/ISCI.2013.6612388

Rangkuti, A.H., R.B. Bahaweres and A. Harjoko, 2012b. Batik image retrieval based on similarity of shape and texture characteristics. Proceedings of the International Conference on Advanced Computer Science and Information Systems, Dec. 1-2, IEEE Xplore Press, Depok, pp: 267-273.

Sanabila, H.R. and R. Manurung, 2009. Recognition of batik motifs using the generalized Hough transform. University of Indonesia.

Wahyudi, A.A., M. Manurung and A. Murni, 2009. Batik image reconstruction based on codebook and keyblock framework. University of Indonesia. 